\title{
Algebraic Structure of Supernilpotent Radical Class Constructed from a Topology Thychonoff Space
}

\author{
Puguh Wahyu Prasetyo $^{1}$, Dian Ariesta Yuwaningsih ${ }^{2}$, Burhanudin Arif Nurnugroho ${ }^{3}$ \\ ${ }^{1,2}$ Universitas Ahmad Dahlan, Indonesia
}

Article Info
Submitted : 23-07-2020
Revised : 25-11-2020
Accepted : 12-10-2020
Published : $17-11-2020$
*Correspondence:
puguh.prasetyo@pmat.uad.ac.id

*Correspondence:
puguh.prasetyo@pmat.uad.ac.id

\begin{abstract}
A radical class of rings is called a supernilpotent radicals if it is hereditary and it contains the class $N_{0}=\left\{A \mid A^{n}=0\right.$ for some positive integer $\left.n\right\}$. In this paper, we start by exploring the concept of Tychonoff space to build a supernilpotent radical. Let $X$ be a Tychonoff space that does not contain any isolated point. The set $C(X)$ of all continuous real-valued functions defined on $X$ is a prime essential ring. Finally, we can show that the class $\bar{L}_{M_{n}(C(X))}$ of rings is a supernilpotent radical class containing the matrix ring $M_{n}(C(X))$.
\end{abstract}

Key Words: supernilpotent radical, Tychonoff space, prime essential rings

http://ejournal.radenintan.ac.id/index.php/al-jabar/index

\section{Introduction}

Topology is one of the branches of Mathematics. Topology is an edge that relates Analysis to Algebra. Since the importance of the Topology materials, the students of Mathematics or Mathematics Education Department should choose the Topology course. Some basic concepts in Topology are important. Topics learned in the Topology course are metric spaces and topological spaces. See the definition and the basic idea of metric space in (Moore \& Cloud, 2007). Furthermore, the explanation of the definition of topological space is below.

Let $X$ be a nonempty set. A collection $\tau$ is called a topological space on $X$ if $\tau$ contains $X$ and the empty set \{\}$, \cup_{i} A_{i} \in \tau$ for every $A_{i}$ where $i$ is an index such that $A_{i} \in \tau$, and the union of these elements satisfies for infinitely many unions or finitely many unions, $\bigcap_{j=1}^{n} A_{j} \in \tau$ for every $A_{j}, j \in\{1,2, \ldots, n\}$, which belongs to $\tau$ (Munkres, 2000).

The collection $\tau=\{X,\{\},\{a\},\{c, d\},\{a, c, d\},\{b, c, d, e, f\}\}$ is one of the topological spaces on the set $X=\{a, b, c, d, e, f\}$. See other examples of topological space in (Munkres, 2000). In this paper, the symbol $R$ denotes the ring with an identity, and the symbol $A$ denotes the ring which does not have an identity. As an introduction to a radical theory of rings, we give the following definition.

A class of rings $\gamma$ is hereditary if for every $A \in \gamma$, then the class of rings $\gamma$ also contains every nonzero $\{0\} \neq I$ ideal of $A$. A class ring $\gamma$ is said to have the inductive property if the ascending chains $I_{1} \subseteq \cdots \subseteq I_{\lambda} \subseteq \cdots$ ideals of $A$ such that $I_{\lambda} \in \gamma$ implies $U I_{\lambda} \in \gamma$. Moreover, a class of rings $\gamma$ is closed under extension if every ring $A$ such that there exists an ideal $I$ of $A$ with $I, A / I \in \gamma$ implies $A \in \gamma$. Furthermore, a class of rings $\gamma$ is said to be closed under homomorphism if $A \in \gamma$, then $A / I \in \gamma$ for every ideal $I$ of $A$. The symbol $I \triangleleft A$ will denote the set $I$ is an ideal of $A$ (Gardner \& Wiegandt, 2004).

On the other hand, a class of rings $\gamma$ is called a radical class of rings if $\gamma$ is closed under homomorphism, $\gamma$ has the inductive property, and $\gamma$ is closed under extension. The definition and some basic properties of the radical class of rings can be seen in (Gardner \& Wiegandt, 
Radical Theory of Rings, 2004). There are two radical class constructions, that are, the lower radical class construction and the upper radical class construction. These two construction types can be learned (Prasetyo, Wahyuni, Wijayanti, \& France-Jackson, 2014). One of the important radical classes used in this article is the prime radical class denoted by $\beta$. The prime radical $\beta$ is the upper radical class $\pi$ of the of all prime rings. Moreover, the prime radical $\beta$ contains the class $N_{0}=\left\{A \mid\right.$ there exists a positive integer $n$ such that $\left.A^{n}=\{0\}\right\}$. The class $N_{0}$ is called the class of all nilpotent rings. A hereditary radical class that contains the class $N_{0}$ of all nilpotent rings is called supernilpotent. In this paper, we give a supernilpotent radical motivated by the existence of the Tychonoff spaces. A Tychonoff space is a Hausdorff space which is complete and regular. Recall the definition of Hausdorff space as follows.

A topological space $X$ is called a Hausdorff space if every couple of points $x_{1}, x_{2}$ which are different in $X$, there exist $U_{1}$ and $U_{2}$ which are the neighborhood of $x_{1}$ and $x_{2}$ respectively such that $U_{1}$ and $U_{2}$ are disjoint (Munkres, 2000).

Every metric space is a Hausdorff space (Munkres, 2000). Hence, the set of all continuous real-valued functions defined on the closed set $[0,1]$, which is denoted by $C[0,1]$ is a Hausdorff space. On the other hand, an ideal $P$ of $A$ is called a prime ideal if there exist ideals $I, J$ of $A$ such that $I J \subseteq P$, implies $I \subseteq P$ or $J \subseteq P$. If $\{0\}$ is a prime ideal of a ring $A$, then the ring $A$ is called a prime ring. Moreover, if every ideal $I$ of $A$ such that $I^{2} \subseteq P$ implies $I \subseteq P$, then the ideal $P$ of $A$ is called a semiprime ideal. If $\{0\}$ is a semiprime ideal of a ring $A$, then $A$ is called a semiprime ring. Finally, the following definition describes the definition of prima essential rings.

A ring $A$ is called a prime essential ring if $R$ is a semiprime ring and for every prime ideal $P$ of $A$ satisfies $P \cap I \neq\{0\}$ for every nonzero ideal $\{0\} \neq I$ of $R$. In other words, every prime ideal of $A$ is essential (Gardner \& Stewart, 1991).

On the other hand, there exist classes of rings which are called special classes. A class $\mu$ of rings is called a special class if $\mu$ is a subclass of the class of all prime rings, $\mu$ is hereditary, and for every $I \in \mu$ such that $A$ is a ring, which is an essential extension of $I$, then $A \in \mu$. A radical class generated as the upper radical of a special class of rings is called a special radical. A special class of rings is also an important topic in the Theory Radical of Rings. The generalization of a special class of rings to a special class of modules was first introduced in (Nicholson \& Watters, 1988). For further results on a special class of modules related to special class of rings constructed by the class of all *-rings can be seen in (Prasetyo, Wijayanti, \& France-Jackson, 2017). Moreover, since the class of all semiprime rings is more general than the class of all prime rings, which is a special class, the existence of a special class of modules motivated the existence of a weakly special class of modules. (Prasetyo, Wijayanti, France-Jackson, \& Repka, 2020) described some properties of a weakly special class of modules. The collection of all supernilpotent radical classes and the collection of all special radical classes form a lattice. The smallest element of the lattice of all supernilpotent radical classes (respectively, all special radical classes) is called a supernilpotent atom (respectively, special atom) (France-Jackson H. , 1987).

The existence of prime essential rings in the development of ring theory, especially in the Radical Theory of Rings, is very important. Described in Theorem 8 in (Gardner \& Stewart, 1991), the set of all prime essential rings will be denoted by $\varepsilon$ and the upper radical of the class of prime essential rings $\mathcal{U}(\varepsilon)$ of $\varepsilon$ is a tool to determine whether any radical class is special or not. Moreover, the existence of prime essential rings in (Wahyuni, Wijayanti, \& France-Jackson, 2017) has been used to construct a special atom, which is not a supernilpotent atom. Hence, the 
prime essential ring is one of the most important rings which should be learned in the Algebra course. In order to make the definition of prime essential rings become clearer, we give an example of prime essential rings.

Example 1. (Gardner \& Stewart, 1991)

Let $\left\{A_{\lambda}, \lambda \in \Lambda\right\}$ be the family of semiprime rings. Then constructed a set $P=\Pi\left\{A_{\lambda} \mid \lambda \in \Lambda\right\}$ and $J=\{p \in P \mid$ such that the cardinality of $\{\lambda \in \Lambda \mid p(\lambda) \neq 0\}$ is less than the cardinality of $\Lambda\}$. Moreover, the factor ring $P / J$ is a prime essential ring.

Example 2. (Wahyuni, WIjayanti, \& France-Jackson, 2017)

Let $\mathbb{Q}$ be the field of all rational numbers. Defined a set $B=\left\{b_{i} \mid i \in \mathbb{Q}\right\}$. In fact, the set $B$ forms a multiplicative semigroup with respect to the binary operation $b_{i} b_{j}=b_{m}$ where $m$ is the maximum of $\{i, j\}$. The semigroup ring $Z_{2}[B]$ defined on $B$ forms a prime essential ring.

As mentioned before, in this paper, we describe a prime essential ring that is motivated by a Tychonoff space. The following proposition described this kind of prime essential ring. The following proposition has been described in (Gardner \& Stewart, 1991) and now we provide the proof since the construction of prime essential ring is one of the fundamental aspect in this paper.

Proposition 3. (Gardner \& Stewart, 1991)

Let $X$ be a Tychonoff space that does not contain any isolated points. Defined a set $C(X)$ on $X$, $C(X)=\{f \mid f$ is a continuous real-valued function defined on $X\}$. Then the set $C(X)$ is a prime essential ring with respect to the additive operation $(f+g)(x)=f(x)+g(x)$ and multiplicative operation $f g(x)=f(x) g(x)$ for every $f, g \in C(X)$.

\section{Proof.}

The first step to prove that $C(X)$ is a prime essential ring; we have to prove that $C(X)$ is a semiprime ring. Let $f, g \in C(X)$ then $(f+g)(x)=f(x)+g(x)$. In fact, $f(x)+g(x)$ is also a continuous real-valued function. Hence, $(f+g)(x)=f(x)+g(x) \in C(X)$. It is clear that the associative property holds for $((f+g)+h)(x)=f(x)+g(x)+h(x)=(f+(g+h))(x)$ for every $f, g, h \in C(X)$. Consider the constant function $f(x)=0$ such that for every $g \in C(X)$ satisfies $(f+g)(x)=(g+f)(x)=g(x)$. Furthermore, for every $f \in C(X)$, there exists $-f \in$ $C(X)$ such that $(f+(-f))(x)=0$. The commutative property of the additive operation also holds. Hence for every $f, g \in C(X)$ satisfies $(f+g)(x)=f(x)+g(x)=g(x)+f(x)=$ $(g+f)(x)$. So, we can infer that $(C(X),+)$ is an abelian group. On the other hand, $f g(x)=$ $f(x) g(x)$ is also a real-valued function which implies $f g(x) \in C(X)$ for every $f, g \in C(X)$. Furthermore, we have the following condition $((f g) h)(x)=f g(x) h(x)=f(x) g(x) h(x)=$ $f(x)(g h(x))(f(g h))(x)$ for every $f, g, h \in C(X)$. The distributive property is held as follows $(f+g) h(x)=((f+g)(x)) h(x)=(f(x)+g(x)) h(x)=f(x) h(x)+g(x) h(x)$ and $f(g+$ $h)(x)=f(x)(g(x)+h(x))=f(x) g(x)+f(x) h(x)$. Hence, we may deduce that $(C(X),+,$. is a ring. It is clear that $C(X)$ is a commutative ring since $f g(x)=f(x) g(x)=g(x) f(x)$ for every $f, g \in C(X)$.

Moreover, we will show that $C(X)$ is a semiprime ring. Let $f \in C(X)$ such that $f C(X) f=$ $\{f g f(x)=0 \mid g \in C(X)\}=\{0\}$. In fact, $f g f(x)=0$ for every $g \in C(X)$. This condition holds if and only if $f(x)=0$. Hence, we can infer that $C(X)$ is a semiprime ring. 
The last step, we will show that $C(X)$ is a prime essential ring. Recall the definition of a prime essential ring is equivalent to Proposition 1 (Gardner \& Stewart, 1991). So, the ring $C(X)$ is a prime essential ring if and only if $C(X)$ does not contain any nonzero ideal, which is a prime ring. Let $\{0\} \neq A \triangleleft C(X)$. Since, $A \neq\{0\}$, there exists $f \in A$ and $x \in X$ such that $f(x) \neq 0$. In fact, the function $f$ is a continuous real-valued function. Then there exists an open neighborhood $\mathcal{N}$ of $x$ such that $z \in \mathcal{N}$ and $f(z) \neq 0$. Since $x$ is not an isolated point, then there exists $w \in \mathcal{N}$ such that $w \neq x$. Since $X$ is a Hausdorff pace, $x$ and $w$ have neighborhoods $\mathcal{N}_{1}$ and $\mathcal{N}_{2}$ respectively, which are disjoint. Furthermore, since $X$ is a Tychonoff space, then there exists $g, h \in C(X)$ such that $g(w)=1$ and $g(z)=0$ for every $z \in X-\mathcal{N}_{1}, h(w)=1$ and $h(z)=0$ for every $z \in X-\mathcal{N}_{2}$. Consider $0 \neq f g$, $f h \in A$ such that $(f g)(f h)=0$. So, we may deduce that $A$ is not a prime ring. Hence, $C(X)$ is a prime essential ring.

\section{The Research Methods}

This research is qualitative research, which conducts a comprehensive literature review on the properties of radical class of rings and prime essential rings. We start by exploring the Hausdorff's property to determine the suitable concept of Hausdorff space in generating a Tychonoff space, which is used to build a prime essential ring. We can found some basic concepts of Hausdorff space (Munkres, 2000).

We scrutinize the property of prime essential rings from the provided reference (Gardner $\&$ Stewart, 1991), and then we claim to clarify that the class of all prime essential ring is close under matrix extension. In a parallel investigation, we develop a prime essential ring which consists of all continuous real-valued function defined on a Tychonoff space. An important role of prime essential rings shown in (Gardner \& Stewart, 1991), (France-Jackson, Wahyuni, \& Wijayanti, 2015), and (France-Jackson H. , 1993).

Finally, we used the constructed prime essential ring to build a supernilpotent radical class and verify whether the assembled prime essential ring becomes a member of that supernilpotent radical class or not.

\section{The Results of the Research and the Discussion}

In this part, we start by giving the following proposition to explain that the set of all $2 \times 2$ matrices such that their entries are members of $C(X)$ forms a prime essential ring.

\section{Proposition 4.}

Let $M_{2}(C(X))=\left\{\left(\begin{array}{ll}f & g \\ h & k\end{array}\right) \mid f, g, h, k \in C(X)\right\}$ be the set of all $2 \times 2$ matrices such that their entries are members of $C(X)$. The set $M_{2}(C(X))$ is a prime essential ring.

\section{Proof.}

It is clear that with respect to matrix addition and matrix multiplication, the set $M_{2}(C(X))$ is a ring. To show that $M_{2}(C(X))$ is a prime essential ring, we have to show that the set $M_{2}(C(X))$ is a free centralizing extension of $C(X)$. Defined a map $\alpha: C(X) \rightarrow M_{2}(C(X))$ as follows:

$$
\alpha(f)=\left(\begin{array}{ll}
f & 0 \\
0 & 0
\end{array}\right)
$$

We will show that $\alpha$ is a monomorphism. It is clear that $\alpha$ is a function. Let $f, g \in C(X)$, we therefore have 


$$
\begin{gathered}
\alpha(f+g)=\left(\begin{array}{cc}
f+g & 0 \\
0 & 0
\end{array}\right)=\left(\begin{array}{cc}
f & 0 \\
0 & 0
\end{array}\right)+\left(\begin{array}{ll}
g & 0 \\
0 & 0
\end{array}\right)=\alpha(f)+\alpha(g) \\
\alpha(f g)=\left(\begin{array}{cc}
f g & 0 \\
0 & 0
\end{array}\right)=\left(\begin{array}{ll}
f & 0 \\
0 & 0
\end{array}\right)\left(\begin{array}{ll}
g & 0 \\
0 & 0
\end{array}\right)=\alpha(f) \alpha(g) .
\end{gathered}
$$

Hence, $\alpha$ is a ring homomorphism. Now let $f, g \in C(X)$ such that $\alpha(f)=\alpha(g)$, then we have

$$
\left(\begin{array}{ll}
f & 0 \\
0 & 0
\end{array}\right)=\left(\begin{array}{ll}
g & 0 \\
0 & 0
\end{array}\right) \Leftrightarrow f=g .
$$

Thus, $\alpha$ is a monomorphism. Furthermore, defined a set

$$
A=\left\{\left(\begin{array}{ll}
f & 0 \\
0 & 0
\end{array}\right) \mid f \in C(X)\right\}
$$

In fact, $A \subset M_{2}(C(X))$ and $C(X) \cong A$ since $A$ is the image of $\alpha$. Hence, $C(X)$ is a subring of $M_{2}(C(X))$ with respect to the embedding process of $C(X)$ over $M_{2}(C(X))$. So $M_{2}(C(X))$ is a free centralizing extension of $C(X)$. It follows from Theorem 2 (Gardner \& Stewart, 1991) that $M_{2}(C(X))$ is a prime essential ring.

\section{Remark 5.}

For further investigation, the property provided in Proposition 3 can be generalized. The set of all $n \times n$ matrices $M_{n}(C(X))$ over $C(X)$ is also a prime essential ring since $M_{n}(C(X))$ is a free centralizing extension of $C(X)$. This condition holds by following Corollary 1 in (Gardner \& Stewart, Prime Essential Ring, 1991).

\section{Example 6.}

It follows from Proposition 3 and Proposition 4 that the following sets are prime essential rings

1. The set $C[0,1]$ of all continuous real-valued functions over a closed interval $[0,1]$.

2. $M_{n}(C[0,1])=\left\{\left(\begin{array}{ll}f & g \\ h & k\end{array}\right) \mid f, g, h, k \in C[0,1]\right\}$

The following corollary will describe the further consequence of the condition described in Proposition 4 and Example 6. But the definition and the concept of the class of rings, which is closed under matrix extension, will be discussed first since the further result related to the closed under matrix extension property.

\section{Definition 7.}

A class $\gamma$ of rings is said to be closed under matrix extension if for every ring $A \in \gamma$ satisfies $M_{n}(A) \in \gamma$, where $M_{n}(A)$ is the set of all $n \times n$ matrices defined on $A$.

\section{Corollary 8}

The set $\rho$ of all prime essential rings is closed under matrix extension.

\section{Proof.}

Let $\rho$ be the class of all prime essential ring. It follows from Corollary 1 (Gardner \& Stewart, 1991) and Example 6 that the set $M_{n}(A)$ of all $n \times n$ matrices defined on $A$, where $A$ is a prime essential ring, is also a prime essential ring. Hence, we can infer that the set $\rho$ of all prime essential rings is closed under matrix extension.

In the next result, we give a supernilpotent radical class construction using the set $C(X)$ of all continuous real-valued functions over a Tychonoff space $X$. As the first step, we explain the definition of an accessible subring. 
Definition 9. (Gardner \& Wiegandt, 2004)

Let $A$ be a ring. A subring $S$ of $A$ is called an $n$-accessible subring if there exist $n$ subrings of $A$, say $S_{1}, S_{2}, \ldots, S_{n}$, such that $S=S_{1} \triangleleft S_{2} \triangleleft \cdots \triangleleft S_{n}=A$. Furthermore, for simplicity, an $n$-accessible subring is called an accessible subring.

\section{Example 10.}

Let $\mathbb{Z}$ be the ring of integers. The set $2 \mathbb{Z}$ is an ideal of $\mathbb{Z}$. Moreover, defined the following sets of matrices.

$$
\begin{gathered}
M_{1}=\left\{\left(\begin{array}{ll}
a & 0 \\
0 & 0
\end{array}\right) \mid a \in 2 \mathbb{Z}\right\} \\
M_{2}=\left\{\left(\begin{array}{cc}
m & 0 \\
0 & n
\end{array}\right) \mid m, n \in 2 \mathbb{Z}\right\} \\
M_{3}=\left\{\left(\begin{array}{ll}
x & 0 \\
0 & y
\end{array}\right) \mid x, y \in \mathbb{Z}\right\}
\end{gathered}
$$

Then, we, therefore, have the following condition

Hence $2 \mathbb{Z} \cong M_{1}$ is an accessible subring of $M_{3}$.

$$
M_{1} \triangleleft M_{2} \triangleleft M_{3}
$$

We will use the existence and the concept of accessible subring as described before to build a supernilpotent radical as follows.

\section{Theorem 11}

Let $C(X)$ be the set of all continuous real-valued functions defined on a set $X$ which is a Tychonoff space, and $X$ does not contain any isolated points. Defined a class of rings $\bar{L}_{M_{n}(C(X))}=$ $\left\{R \mid R \cong S / K\right.$ for some accessible subrings $S$ of $\left.M_{n}(C(X))\right\} \cup \beta$ where $\beta$ is the prime radical class. The class $\bar{L}_{M_{n}(C(X))}$ is a supernilpotent radical class.

\section{Proof.}

The first step, we will show that $\bar{L}_{M_{n}(C(X))}$ is not an empty set. Since $\beta \subset \bar{L}_{M_{n}(C(X))}$, we can infer that $\bar{L}_{M_{n}(C(X))} \neq\{\}$ and $\bar{L}_{M_{n}(C(X))}$ contains the set $N_{0}$ of all nilpotent rings because the prime radical class $\beta$ contains $N_{0}$. Moreover, we will show that for every $\operatorname{ring} R \in \bar{L}_{M_{n}(C(X))}$, then $I \in$ $\bar{L}_{M_{n}(C(X))}$ for every ideal $I$ of $R$. Let $R \in \bar{L}_{M_{n}(C(X))}$, it follows from the construction of $\bar{L}_{M_{n}(C(X))}$ that $\bar{L}_{M_{n}(C(X))}$ is a lower radical class which is hereditary and it is closed under homomorphism containing the matrix ring $M_{n}(C(X))$. By Corollary 3.2.10 (Gardner \& Stewart, 1991), we may deduce that $I \in \bar{L}_{M_{n}(C(X))}$. Thus $\bar{L}_{M_{n}(C(X))}$ is a supernilpotent radical class.

Finally, we also have the following theorem as our final result.

\section{Theorem 12}

The prime essential ring $C(X) \in \bar{L}_{M_{n}(C(X))}$.

\section{Proof.}

Consider the set of matrices as follows

$$
S=\left\{\left(\begin{array}{ll}
f & 0 \\
0 & 0
\end{array}\right) \mid f \in C(X)\right\}
$$

In fact, it is clear that the set $S$ is an accessible subring of $M_{n}(C(X))$ such that $S \cong C(X)$. The special case for $M_{2}(C(X))$ is explained in Proposition 4. Hence, $C(X)$ is a homomorphic image of $S$ and $S$ is an accessible subring of $M_{n}(C(X))$. So, we can infer that $C(X) \in \bar{L}_{M_{n}(C(X))}$. 


\section{Conclusion and Suggestion}

Based on our investigation on the property of prime essential rings, we can infer that the class of all prime essential rings is closed under matrix extension. Furthermore, we can construct the prime essential ring $C(X)$ which consists of all continuous real-valued functions defined on the Tychonoff space $X$. We also show that the set $M_{n}(C(X))$ of all $n \times n$ over $C(X)$ is also a prime essential ring. Finally, we can show that $\bar{L}_{M_{n}(C(X))}$ is a supernilpotent radical class containing the ring $C(X)$. For further investigation, we suggest to the reader to explore the property of $\bar{L}_{M_{n}(C(X))}$ and determine whether the supernilpotent radical $\bar{L}_{M_{n}(C(X))}$ is an atom of the lattice of all supernilpoten radical or not.

\section{Acknowledgment}

The authors express gratitude for the Mathematics Education Department, Universitas Ahmad Dahlan, and Lembaga Penelitian dan Pengabdian Kepada Masyarakat (LPPM) Universitas Ahmad Dahlan for the financial support of this research under fundamental research grant PD-120/SP3/LPPM-UAD/2020. The authors would like to thank Dr. Ahmad Faisol, M.Sc from the Mathematics Department, Universitas Lampung, for the comments and suggestions in the preparation of this paper. The authors also thank to the referee for the contructive comments and suggestions.

\section{References}

France-Jackson, H. (1987). On Atoms of The Lattice of Supernilpotent Radicals. Quaestiones Mathematicae, 251-255.

France-Jackson, H. (1993). On Prime Essential Rings. Bull. Austral. Math. Soc, 287-290.

France-Jackson, H., Wahyuni, S., \& Wijayanti, I. E. (2015). Radical Related To Special Atoms Revisited. Bulletin of Australian Mathematical Society, 202-210.

Gardner, B. J., \& Stewart, P. M. (1991). Prime Essential Rings. Proceedings of The Edinburgh Mathematical Society, 241-250.

Gardner, B. J., \& Stewart, P. N. (1991). Prime Essential Ring. Proceedings of the Edinburgh Mathematical Society, 241-250.

Gardner, B. J., \& Wiegandt, R. (2004). Radical Theory of Rings. New York: Marcel Dekker, Inc.

Moore, R. E., \& Cloud, M. J. (2007). Computational Functional Analysis. Cambridge: Woodhead Publishing.

Munkres, J. R. (2000). Topology. New Jersey: Prentice Hall.

Nicholson, W. K., \& Watters, J. F. (1988). Normal Radicals and Normal Classes of. Glasgow Math. J., 97-100.

Prasetyo, P. W., Wahyuni, S., Wijayanti, I. E., \& France-Jackson, H. (2014). Dari Radikal Ring Ke Radikal Modul. Prosiding Seminar Nasional Matematika (pp. 272-282). Jember: Universitas Jember.

Prasetyo, P. W., Wijayanti, I. E., \& France-Jackson, H. (2017). *p-Modues and A Special Class of Modules Determined by The Essential Class of Modules Determined By The Essential Closure of The Class of All *-Rings. JP Journal of Algebra, Number Theory and Applications, 11-20. 
Prasetyo, P. W., Wijayanti, I. E., France-Jackson, H., \& Repka, J. (2020). Weakly Special Class of Modules. Mathematics and Statistics, 23-27.

Wahyuni, S., Wijayanti, I. E., \& France-Jackson, H. (2017). A Prime Essential Ring That Generates A Special Atom. Bulletin of The Australian Mathematical Society, 214-2018. 